\title{
A modified kernel method for a time-fractional inverse diffusion problem
}

Songshu Liu' ${ }^{1}$ and Lixin Feng ${ }^{2 *}$

${ }^{\text {"Correspondence: }}$

fenglixin@hlju.edu.cn

${ }^{2}$ School of Mathematical Sciences, Heilongjiang University, Harbin,

150080, China

Full list of author information is

available at the end of the article

\begin{abstract}
In this paper, we consider a time-fractional inverse diffusion problem, where the data is given at $x=1$ and the solution is sought in the interval $0 \leq x<1$. Such a problem is obtained from the classical diffusion equation by replacing the first-order time derivative by the Caputo fractional derivative of order $\alpha \in(0,1)$. We show that a time-fractional inverse diffusion problem is severely ill-posed and we further apply a modified kernel method to solve it based on the solution in the frequency domain. The corresponding convergence estimates are provided. Finally, an example is constructed to show the feasibility and efficiency of the proposed method.
\end{abstract}

MSC: 35R25; 35R30; 47A52

Keywords: time-fractional inverse diffusion problem; ill-posed problem; regularization method; error estimate

\section{Introduction}

In the past decades, studies on the problems of the partial differential equation mainly focused on direct problems and inverse problems of integer order differential equation, and some numerical techniques have been proposed to solve integer order differential equation [1-8]. However, fractional derivatives calculus and fractional differential equations have been used recently to solve a range of problems in mechanical engineering [9], viscoelasticity [10], electron transport [11], dissipation [12], heat conduction [13, 14], and high-frequency financial data [15].

The time-fractional diffusion equation arises by replacing the standard time partial derivative in the diffusion equation with a time-fractional partial derivative. It is usually used to describe the anomalous diffusion (superdiffusion, non-Gaussian diffusion, subdiffusion) which is not consistent with the classical Fick (or Fourier) law [16]. The direct problem, i.e., initial value problem and initial boundary for time-fractional diffusion equation have been studied extensively in the past few years [17-22]. However, in some practical problems, the boundary data on the whole boundary cannot be obtained. We only know the noisy data on a part of the boundary or at some interior points of the concerned domain. This leads to an inverse and ill-posed problem of the fractional diffusion equation, which means the solution does not depend continuously on the given known conditions. In this paper, we investigate an inverse problem of the time-fractional diffusion equation. This kind of ill-posed problem is important in many branches of engineering sciences $[23,24]$.

(c) 2015 Liu and Feng. This article is distributed under the terms of the Creative Commons Attribution 4.0 International License (http://creativecommons.org/licenses/by/4.0/), which permits unrestricted use, distribution, and reproduction in any medium, provided you give appropriate credit to the original author(s) and the source, provide a link to the Creative Commons license, and indicate if changes were made. 
Due to the difficulty of the fractional derivative and the ill-posedness, to the authors' knowledge, the results on inverse problem of the time-fractional diffusion equation are very few. The uniqueness of an inverse problem for a one-dimensional fractional diffusion equation was given in [25]. Cheng and $\mathrm{Fu}[26]$ gave an iteration regularization method for a time-fractional inverse diffusion problem. Zheng and Wei $[27,28]$ investigated a time-fractional inverse diffusion problem by using a spectral regularization method and a modified equation method.

In this article, we consider the following time-fractional inverse diffusion problem (TFIDP):

$$
\begin{cases}-u_{x}(x, t)={ }_{0} D_{t}^{\alpha} u(x, t), & x>0, t>0,0<\alpha<1 \\ u(x, 0)=0, & x \geq 0, \\ u(1, t)=f(t), & t \geq 0, \\ \lim _{x \rightarrow \infty} u(x, t)=0, & t \geq 0\end{cases}
$$

where the time-fractional derivative ${ }_{0} D_{t}^{\alpha} u(x, t)$ is the Caputo fractional derivative of order $\alpha \in(0,1)$ defined by [29]

$$
\begin{aligned}
& { }_{0} D_{t}^{\alpha} u(x, t)=\frac{1}{\Gamma(1-\alpha)} \int_{0}^{t} \frac{\partial u(x, s)}{\partial s} \frac{d s}{(t-s)^{\alpha}}, \quad 0<\alpha<1, \\
& { }_{0} D_{t}^{\alpha} u(x, t)=\frac{\partial u(x, t)}{\partial t}, \quad \alpha=1,
\end{aligned}
$$

where $\Gamma(\cdot)$ is the gamma function.

The TFIDP is an inverse problem and is severely ill-posed. That means the solution does not depend continuously on the given data, and any small perturbation in the given data may cause large changes to the solution. In this paper, we will present a modified kernel method to construct a stable approximation solution of the TFIDP.

The rest of this paper is organized as follows. In Section 2, we demonstrate ill-posedness of the time-fractional inverse diffusion problem. In Section 3, a modified kernel method is used to solve problem (1), and we also obtain the convergence estimates between the regularization solution and the exact solution based on the $a$ priori assumptions for the exact solution. In Section 4, an example is illustrated to show the main results. Finally, we conclude this paper in Section 5.

\section{III-posedness of a time-fractional inverse diffusion problem}

In order to apply the Fourier analysis of the time-fractional inverse diffusion problem, we extend all the functions to the whole line $-\infty<t<\infty$ by defining them to be zero for $t<0$ whenever it is necessary. We also assume all the functions involving $t$ variable are in $L^{2}(\mathbb{R})$. Here, and in the following sections, we use the corresponding $L^{2}$ norm, as defined below:

$$
\|g\|=\left(\int_{-\infty}^{\infty}|g(t)|^{2} d t\right)^{\frac{1}{2}} .
$$

Let

$$
\hat{g}(\xi)=\frac{1}{\sqrt{2 \pi}} \int_{-\infty}^{\infty} g(t) e^{-i \xi t} d t
$$


be the Fourier transform of the function $g(t) \in L^{2}(\mathbb{R})$ and $\|\cdot\|_{p}$ denote the $H_{p}$ norm, i.e.,

$$
\|g\|_{p}=\left(\int_{-\infty}^{\infty}\left(1+\xi^{2}\right)^{p}|\hat{g}(\xi)|^{2} d \xi\right)^{\frac{1}{2}} .
$$

Then, applying the Fourier transforming with respect to $t$ to problem (1), we have [29]

$$
\begin{aligned}
& \hat{u}_{x}(x, \xi)=-(i \xi)^{\alpha} \hat{u}(x, \xi), \\
& \hat{u}(1, \xi)=\hat{g}(\xi) .
\end{aligned}
$$

The solution of the above problem can be given by

$$
\hat{u}(x, \xi)=e^{l(\xi)(1-x)} \hat{g}(\xi)
$$

where

$$
l(\xi)=(i \xi)^{\alpha}= \begin{cases}|\xi|^{\alpha}\left(\cos \frac{\alpha \pi}{2}+i \sin \frac{\alpha \pi}{2}\right), & \xi \geq 0, \\ |\xi|^{\alpha}\left(\cos \frac{\alpha \pi}{2}-i \sin \frac{\alpha \pi}{2}\right), & \xi<0 .\end{cases}
$$

For the above problem, note that $l(\xi)$ has a positive real part $|\xi|^{\alpha} \cos \frac{\alpha \pi}{2}$, the small error in the high-frequency components will be amplified by the factor $e^{|\xi|^{\alpha} \cos \frac{\alpha \pi}{2}(1-x)}$. Therefore the TFIDP for recovering the temperature $u(x, t)$ from the measured data $g^{\delta}(t)$ is severely ill-posed. Here, suppose that the measured data $g^{\delta}(t) \in L^{2}(\mathbb{R})$ satisfy

$$
\left\|g^{\delta}(t)-g(t)\right\| \leq \delta
$$

where the constant $\delta>0$ is the noise level.

To solve the problem (1), a natural way to stabilize the problem is to eliminate the high frequencies or to replace the 'kernel' $e^{l(\xi)(1-x)}$ by a bounded approximation.

We now list two kernels of regularization methods for solving the time-fractional inverse diffusion problem.

The first is

$$
e^{(i \xi)^{\alpha}(1-x)} \chi_{\max },
$$

where $\chi_{\max }$ denotes the characteristic function of interval $\left[-\xi_{\max }, \xi_{\max }\right]$, and $\xi_{\max }$ is a regularization parameter. It corresponds to a spectral regularization method; see [27].

The second is

$$
e^{\frac{(i \xi)^{\alpha}}{1+\mu \xi^{2}}(1-x)},
$$

where $\mu$ is a regularization parameter. It corresponds to a modified equation method; see [28].

In this article, we propose a regularization method by modifying the 'kernel' to deal with the difficulty of problem (1) as follows:

$$
\frac{e^{l(\xi)(1-x)}}{1+\beta^{2} e^{2|\xi|^{\alpha} \cos \frac{\alpha \pi}{2}}},
$$

where $\beta$ is a regularization parameter. 
Now, we will apply this regularization strategy to solve a time-fractional inverse diffusion problem and prove that this regularization strategy is feasible.

\section{A modified kernel method and error estimates}

In this section, we will construct regularization solution by modifying the 'kernel' and obtain convergence estimates.

Here, we give an approximate solution of problem (1) by perturbing the kernel of (6) as follows:

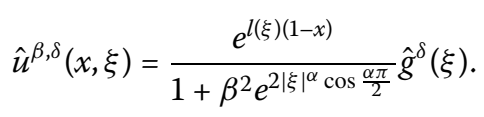

In order to obtain our main results, we first give two important lemmas. Here, we set $\eta=|\xi|^{\alpha} \cos \frac{\alpha \pi}{2}$.

Lemma 1 If $0<x<1,0<\alpha<1$, then we have

$$
\sup _{\eta \geq 0} \frac{e^{\eta(1-x)}}{1+\beta^{2} e^{2 \eta}} \leq \beta^{x-1} .
$$

Proof Let

$$
F(\eta)=\frac{e^{\eta(1-x)}}{1+\beta^{2} e^{2 \eta}}
$$

by elementary calculation, we see that $\eta=\frac{1}{2} \ln \left(\frac{1-x}{1+x} \beta^{-2}\right)$ is the maximum point of $F(\eta)$. So, we know

$$
\left(1+\beta^{2} e^{2 \eta}\right)^{-1}=\left(1+\frac{1-x}{1+x}\right)^{-1}=\left(\frac{2}{1+x}\right)^{-1}=\frac{1+x}{2} \leq 1
$$

and

$$
e^{\eta(1-x)}=\left(\frac{1-x}{1+x} \beta^{-2}\right)^{\frac{1-x}{2}}=\left(\frac{1-x}{1+x}\right)^{\frac{1-x}{2}} \beta^{x-1} \leq \beta^{x-1} .
$$

Combining (10) and (11), we have

$$
\sup _{\eta \geq 0} \frac{e^{\eta(1-x)}}{1+\beta^{2} e^{2 \eta}} \leq \beta^{x-1}
$$

Lemma 2 If $0<x<1,0<\alpha<1$, then we have

$$
\sup _{\eta \geq 0} \frac{e^{\eta(2-x)}}{1+\beta^{2} e^{2 \eta}} \leq \beta^{x-2}
$$

Proof The proof of this lemma is similar to that of Lemma 1, we get the inequality (12).

In the following theorems, the convergence estimates between the regularization solution and the exact solution will be given based on an a priori choice of the regularization parameter. 
Theorem 1 Suppose that $u^{\beta, \delta}(x, t)$ is the regularization solution for problem (1) with noisy data $g^{\delta}(t)$ and that $u(x, t)$ is the exact solution for problem (1) with the exact data $g(t)$. Let the assumption $\left\|g^{\delta}(t)-g(t)\right\| \leq \delta$ be satisfied and let $\|u(0, \cdot)\| \leq E$ hold. If we choose

$$
\beta=\frac{\delta}{E}
$$

then for every $x \in(0,1)$, we obtain the following error estimate:

$$
\left\|u^{\beta, \delta}(x, \cdot)-u(x, \cdot)\right\| \leq 2 \delta^{x} E^{1-x}
$$

Proof By Parseval's identity and the triangle inequality, we have

$$
\begin{aligned}
& \left\|u^{\beta, \delta}(x, \cdot)-u(x, \cdot)\right\|=\left\|\hat{u}^{\beta, \delta}(x, \cdot)-\hat{u}(x, \cdot)\right\| \\
& =\left\|\frac{e^{l(\xi)(1-x)}}{1+\beta^{2} e^{2|\xi|^{\alpha} \cos \frac{\alpha \pi}{2}}} \hat{g}^{\delta}(\xi)-e^{l(\xi)(1-x)} \hat{g}(\xi)\right\|
\end{aligned}
$$

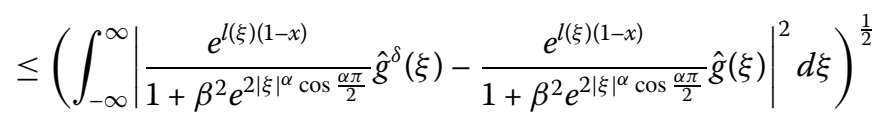

$$
\begin{aligned}
& +\left(\int_{-\infty}^{\infty}\left|\frac{e^{l(\xi)(1-x)}}{1+\beta^{2} e^{2|\xi|^{\alpha} \cos \frac{\alpha \pi}{2}}} \hat{g}(\xi)-e^{l(\xi)(1-x)} \hat{g}(\xi)\right|^{2} d \xi\right)^{\frac{1}{2}}
\end{aligned}
$$

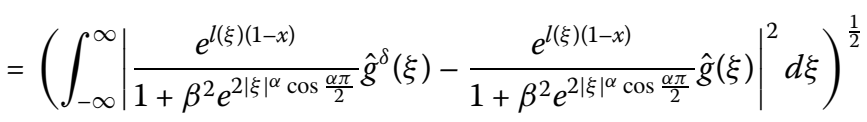

$$
\begin{aligned}
& +\left(\int_{-\infty}^{\infty}\left|\left(\frac{e^{-l(\xi) x}}{1+\beta^{2} e^{2|\xi|^{\alpha} \cos \frac{\alpha \pi}{2}}}-e^{-l(\xi) x}\right) e^{l(\xi)} \hat{g}(\xi)\right|^{2} d \xi\right)^{\frac{1}{2}} \\
& \leq \delta \sup _{\xi \in \mathbb{R}} \frac{\left|e^{l(\xi)(1-x)}\right|}{1+\beta^{2} e^{2|\xi|^{\alpha} \cos \frac{\alpha \pi}{2}}} \\
& +\sup _{\xi \in \mathbb{R}}\left|\frac{\beta^{2} e^{(2-x)|\xi|^{\alpha} \cos \frac{\alpha \pi}{2}-i x \operatorname{sign}(\xi)|\xi|^{\alpha} \sin \frac{\alpha \pi}{2}}}{1+\beta^{2} e^{2|\xi|^{\alpha} \cos \frac{\alpha \pi}{2}}}\right|\|\hat{u}(0, \cdot)\| \\
& \leq \delta \sup _{\xi \in \mathbb{R}} K_{1}(\xi)+E \sup _{\xi \in \mathbb{R}} K_{2}(\xi),
\end{aligned}
$$

where

$$
\begin{aligned}
& K_{1}(\xi)=\frac{e^{|\xi|^{\alpha} \cos \frac{\alpha \pi}{2}(1-x)}}{1+\beta^{2} e^{2|\xi|^{\alpha} \cos \frac{\alpha \pi}{2}}}, \\
& K_{2}(\xi)=\frac{\beta^{2} e^{(2-x)|\xi|^{\alpha} \cos \frac{\alpha \pi}{2}}}{1+\beta^{2} e^{2|\xi|^{\alpha} \cos \frac{\alpha \pi}{2}}} .
\end{aligned}
$$

Combining (9) and (12), and setting $\eta=|\xi|^{\alpha} \cos \frac{\alpha \pi}{2}$, we get

$$
\begin{aligned}
& K_{1}(\xi)=\frac{e^{\eta(1-x)}}{1+\beta^{2} e^{2 \eta}} \leq \beta^{x-1}, \\
& K_{2}(\xi)=\frac{\beta^{2} e^{(2-x) \eta}}{1+\beta^{2} e^{2 \eta}} \leq \beta^{2} \beta^{x-2}=\beta^{x} .
\end{aligned}
$$


Therefore,

$$
\left\|u^{\beta, \delta}(x, \cdot)-u(x, \cdot)\right\| \leq \delta \beta^{x-1}+E \beta^{x},
$$

according to (13), we can get the error estimate

$$
\left\|u^{\beta, \delta}(x, \cdot)-u(x, \cdot)\right\| \leq 2 \delta^{x} E^{1-x}
$$

The error estimate in Theorem 1 does not give any useful information on the continuous dependence of the solution at $x=0$. To retain the continuous dependence of the solution at $x=0$, one has to introduce a stronger a priori assumption.

We are now in the position to formulate the convergence rate for $x=0$.

Theorem 2 Suppose that $u^{\beta, \delta}(x, t)$ is the regularization solution for problem (1) with noisy data $g^{\delta}(t)$ and that $u(x, t)$ is the exact solution for problem (1) with the exact data $g(t)$. Let the assumption $\left\|g^{\delta}(t)-g(t)\right\| \leq \delta$ be satisfied and let $\|u(0, \cdot)\|_{p} \leq E(p \geq 1)$ hold. If we choose

$$
\beta=\left(\frac{\delta}{E}\right)^{\gamma}, \quad \frac{1}{2}<\gamma<1
$$

then, for $x=0$, we obtain the following error estimate:

$$
\begin{aligned}
& \left\|u^{\beta, \delta}(0, \cdot)-u(0, \cdot)\right\| \\
& \leq \delta^{1-\gamma} E^{\gamma}+\sqrt{\pi} \delta^{2 \gamma-\cos \frac{\alpha \pi}{2}} E^{1-2 \gamma+\cos \frac{\alpha \pi}{2}}\left(\ln \frac{E}{\delta}\right)^{-p \cos \frac{\alpha \pi}{2}} \\
& \quad+E\left[\frac{1}{2} \ln \left(\frac{E}{\delta}\left(\ln \frac{E}{\delta}\right)^{-p}\right)\right]^{-\frac{p}{\alpha}} \cdot
\end{aligned}
$$

Proof It is similar to Theorem 1; we obtain

$$
\begin{aligned}
& \left\|u^{\beta, \delta}(x, \cdot)-u(x, \cdot)\right\| \\
& \leq\left(\int_{-\infty}^{\infty}\left|\frac{e^{l(\xi)(1-x)}}{1+\beta^{2} e^{2|\xi|^{\alpha} \cos \frac{\alpha \pi}{2}}}\left(\hat{g}^{\delta}(\xi)-\hat{g}(\xi)\right)\right|^{2} d \xi\right)^{\frac{1}{2}} \\
& +\left(\int_{-\infty}^{\infty}\left|\frac{\beta^{2} e^{(2-x)|\xi|^{\alpha} \cos \frac{\alpha \pi}{2}-i x \operatorname{sign}(\xi)|\xi|^{\alpha} \sin \frac{\alpha \pi}{2}}}{\left(1+\beta^{2} e^{2|\xi|^{\alpha} \cos \frac{\alpha \pi}{2}}\right)\left(1+\xi^{2}\right)^{\frac{p}{2}}}\left(1+\xi^{2}\right)^{\frac{p}{2}} \hat{u}(0, \cdot)\right|^{2} d \xi\right)^{\frac{1}{2}} \\
& =K_{3}(\xi)+K_{4}(\xi) \text {. }
\end{aligned}
$$

Setting $\eta=|\xi|^{\alpha} \cos \frac{\alpha \pi}{2}$, and combining (9) and (12), we get

$$
K_{3}(\xi) \leq \delta \sup _{\eta \in R} \frac{e^{\eta(1-x)}}{1+\beta^{2} e^{2 \eta}} \leq \delta \beta^{x-1}
$$


and

$$
\begin{aligned}
K_{4}(\xi)= & \left(\int_{-\infty}^{\infty}\left|\frac{\beta^{2} e^{(2-x) \eta}}{\left(1+\beta^{2} e^{2 \eta}\right)\left(1+\xi^{2}\right)^{\frac{p}{2}}}\left(1+\xi^{2}\right)^{\frac{p}{2}} \hat{u}(0, \cdot)\right|^{2} d \xi\right)^{\frac{1}{2}} \\
\leq & E\left(\int_{|\xi| \leq \xi_{0}}\left[\frac{\beta^{2} e^{(2-x) \eta}}{\left(1+\beta^{2} e^{2 \eta}\right)\left(1+\xi^{2}\right)^{\frac{p}{2}}}\right]^{2} d \xi\right)^{\frac{1}{2}} \\
& +E\left(\int_{|\xi|>\xi_{0}}\left[\frac{\beta^{2} e^{(2-x) \eta}}{\left(1+\beta^{2} e^{2 \eta}\right)\left(1+\xi^{2}\right)^{\frac{p}{2}}}\right]^{2} d \xi\right)^{\frac{1}{2}} \\
\leq & E \sqrt{\pi} \beta^{2}\left(\left.e^{(2-x) \eta_{0}}\right|_{\eta_{0}=\left|\xi_{0}\right|^{\alpha} \cos \frac{\alpha \pi}{2}}\right)+E \beta^{x} \xi_{0}^{-p} .
\end{aligned}
$$

Taking $\xi_{0}=\left[\frac{1}{2} \ln \left(\frac{E}{\delta}\left(\ln \frac{E}{\delta}\right)^{-p}\right)\right]^{\frac{1}{\alpha}}$ and using (15), we get

$$
\begin{aligned}
& \left\|u^{\beta, \delta}(x, \cdot)-u(x, \cdot)\right\| \\
& \leq \delta \beta^{x-1}+\sqrt{\pi} E \beta^{2}\left(\left.e^{(2-x) \eta_{0}}\right|_{\eta_{0}=\left|\xi_{0}\right|^{\alpha} \cos \frac{\alpha \pi}{2}}\right)+E \beta^{x} \xi_{0}^{-p} \\
& \leq \delta^{1-\gamma+\gamma x} E^{\gamma-\gamma x}+\sqrt{\pi} \delta^{2 \gamma-\left(1-\frac{x}{2}\right) \cos \frac{\alpha \pi}{2}} E^{1-2 \gamma+\left(1-\frac{x}{2}\right) \cos \frac{\alpha \pi}{2}}\left(\ln \frac{E}{\delta}\right)^{\left(\frac{p x}{2}-p\right) \cos \frac{\alpha \pi}{2}} \\
& \quad+\delta^{\gamma x} E^{1-\gamma x}\left[\frac{1}{2} \ln \left(\frac{E}{\delta}\left(\ln \frac{E}{\delta}\right)^{-p}\right)\right]^{-\frac{p}{\alpha}} .
\end{aligned}
$$

Here, for $x=0$, we obtain

$$
\begin{aligned}
& \left\|u^{\beta, \delta}(0, \cdot)-u(0, \cdot)\right\| \\
& \leq \delta^{1-\gamma} E^{\gamma}+\sqrt{\pi} \delta^{2 \gamma-\cos \frac{\alpha \pi}{2}} E^{1-2 \gamma+\cos \frac{\alpha \pi}{2}}\left(\ln \frac{E}{\delta}\right)^{-p \cos \frac{\alpha \pi}{2}} \\
& \quad+E\left[\frac{1}{2} \ln \left(\frac{E}{\delta}\left(\ln \frac{E}{\delta}\right)^{-p}\right)\right]^{-\frac{p}{\alpha}} \cdot
\end{aligned}
$$

\section{Numerical example}

The purpose of this section is to present a numerical example and illustrate the accuracy and efficiency of the proposed method. Here, the proposed methods will be implemented in Matlab. In our numerical experiment, we fix the interval $0 \leq t \leq 1$.

To illustrate the behavior of a modified kernel regularization method, we construct the test problems with a given function $f$ at $x=0$, then we compute a data function $g$ at $x=1$ according to $\hat{g}(\xi)=e^{-l(\xi)} \hat{f}(\xi)$, which is well-posed. We usually think that the computed data $g$ is exact. The discrete noisy data $g^{\delta}$ is obtained by adding a random noise to the exact data $g$, that is,

$$
g^{\delta}=g+\varepsilon \operatorname{randn}(\operatorname{size}(g))
$$

where

$$
g=\left(g\left(x_{1}\right), g\left(x_{2}\right), \ldots, g\left(x_{n}\right)\right)^{T}, \quad x_{i}=(i-1) \Delta x, \Delta x=\frac{1}{n-1}, i=1,2, \ldots, n .
$$


Then the total noise $\delta$ can be measured in the sense of the root mean square error according to

$$
\delta:=\left\|g^{\delta}-g\right\|_{l^{2}}=\sqrt{\frac{1}{n} \sum_{i=1}^{n}\left(g_{i}^{\delta}-g_{i}\right)^{2}} .
$$

Here, the function $\operatorname{randn}(\cdot)$ generates arrays of random numbers whose elements are normally distributed with mean 0 , variance $\sigma^{2}=1$ and standard deviation $\sigma=1$, the function $\operatorname{randn}(\operatorname{size}(g))$ returns an array of random entries that has the same size as $g$.

Example 1 Consider a smooth function

$$
f(t)= \begin{cases}\frac{1}{t^{3 / 2}} e^{-\frac{1}{4 t}}, & t>0, \\ 0, & \text { others }\end{cases}
$$

The comparison of the computational effects with $\alpha=0.3, \varepsilon=0.001$, at $x=0.8,0.5,0.2,0$ are shown in Figure 1.

The comparison of the computational effects with $\alpha=0.4, x=0$, for $\varepsilon=0.00001,0.0001$, 0.001, 0.01 are shown in Figure 2.

The comparison of the computational effects with $\alpha=0.6, x=0, \varepsilon=0.0001$ for $\gamma=$ 0.6, 0.7, 0.8, 0.9 are shown in Figure 3.

From Figures 1-3 (see also Tables 1-3), we can find that the numerical results near the boundary $x=1$ are better than the ones close to $x=0$, and the smaller the parameter $\varepsilon$,

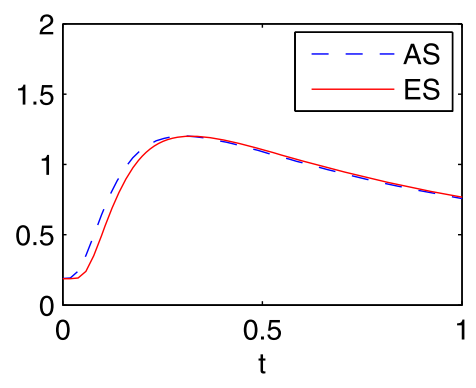

(a)

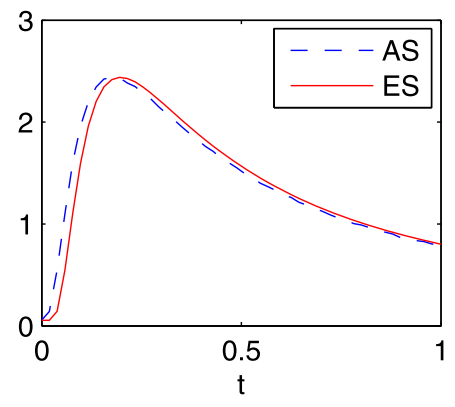

(c)

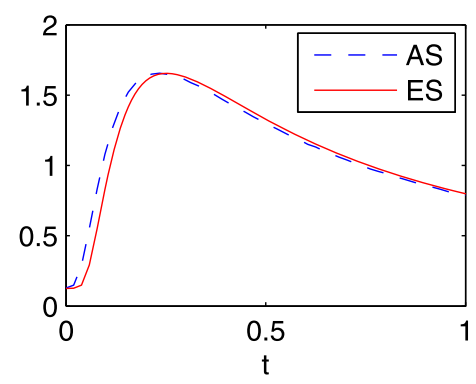

(b)

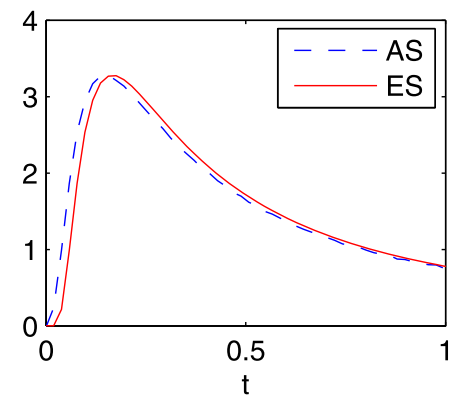

(d)

Figure 1 The exact solution (ES) and its approximation solution (AS). $\alpha=0.3, \varepsilon=0.001$ and (a) $x=0.8$; (b) $x=0.5$; (c) $x=0.2$; (d) $x=0$. 


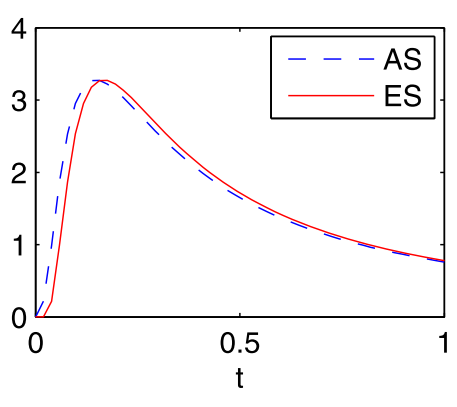

(a)

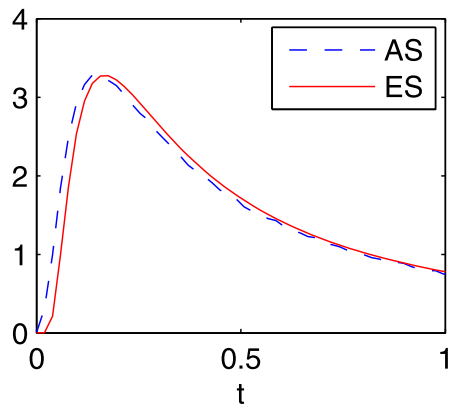

(c)

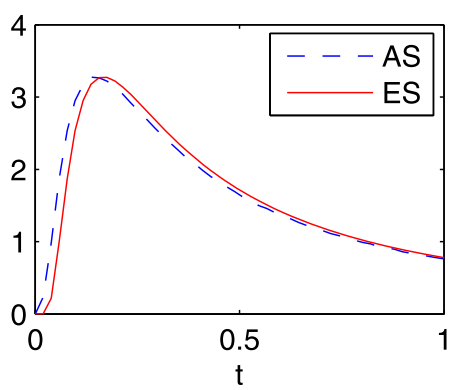

(b)

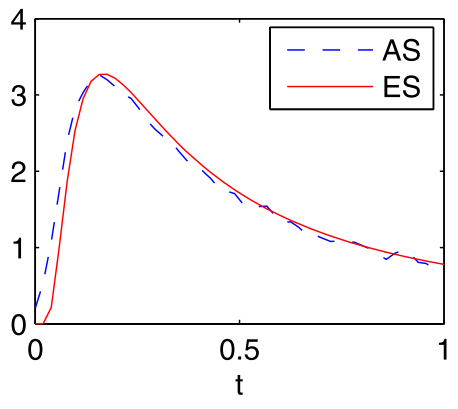

(d)

Figure 2 The exact solution (ES) and its approximation solution (AS). $\alpha=0.4, x=0$ and (a) $\varepsilon=0.00001$; (b) $\varepsilon=0.0001$; (c) $\varepsilon=0.001$; (d) $\varepsilon=0.01$.

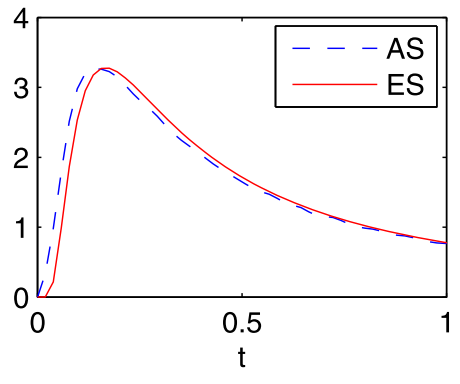

(a)

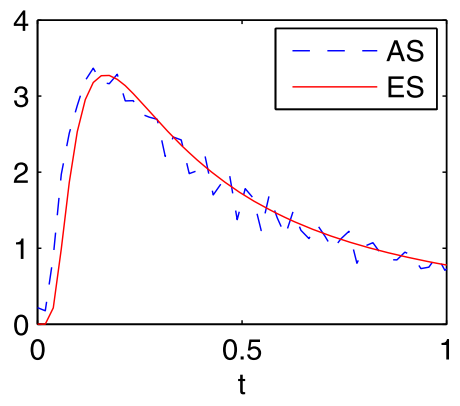

(c)

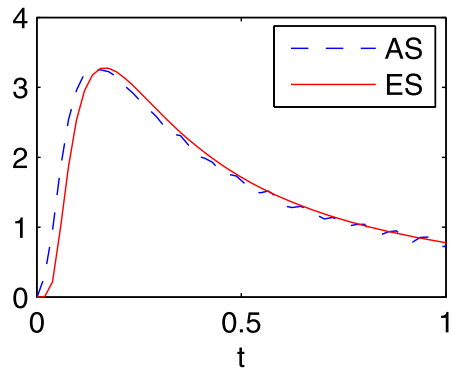

(b)

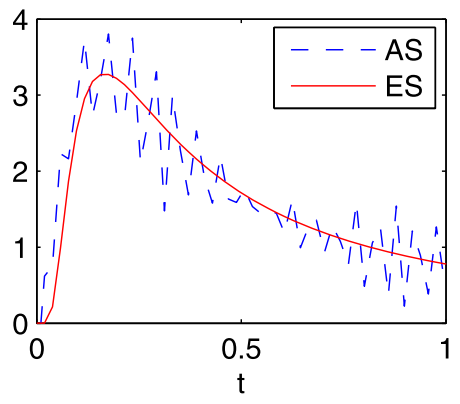

(d)

Figure 3 The exact solution (ES) and its approximation solution (AS). $\alpha=0.6, x=0$ and (a) $\gamma=0.6$; (b) $\gamma=0.7$; (c) $\gamma=0.8$; (d) $\gamma=0.9$. 
Table 1 The executing time for the running time program with different $x$ for Example 1

\begin{tabular}{lllll}
\hline $\boldsymbol{x}$ & $\mathbf{0 . 8}$ & $\mathbf{0 . 5}$ & $\mathbf{0 . 2}$ & $\mathbf{0}$ \\
\hline$t$ & 9.420000 & 9.526000 & 9.483000 & 9.389000 \\
\hline
\end{tabular}

Table 2 The executing time for the running time program with different $\varepsilon$ for Example 1

\begin{tabular}{lllll}
\hline $\boldsymbol{\varepsilon}$ & $\mathbf{0 . 0 0 0 0 1}$ & $\mathbf{0 . 0 0 0 1}$ & $\mathbf{0 . 0 0 1}$ & $\mathbf{0 . 0 1}$ \\
\hline$t$ & 9.241000 & 9.494000 & 9.531000 & 9.546000 \\
\hline
\end{tabular}

Table 3 The executing time for the running time program with different $\gamma$ for Example 1

\begin{tabular}{lllll}
\hline $\boldsymbol{\gamma}$ & $\mathbf{0 . 6}$ & $\mathbf{0 . 7}$ & $\mathbf{0 . 8}$ & $\mathbf{0 . 9}$ \\
\hline$t$ & 9.346000 & 9.508000 & 9.514000 & 9.523000 \\
\hline
\end{tabular}

the better the computed approximation is. Moreover, for $x=0$, we can see that the smaller the parameter $\gamma$, the better the computed approximation is.

\section{Conclusion}

In this paper, we propose a modified kernel method for solving time-fractional inverse diffusion problem by producing a stable approximation solution. For this regularization strategy, in the presence of noisy data, we establish and prove the convergence estimates for the cases $0 \leq x<1$ under the $a$ priori bound assumptions for the exact solution and the suitable choices of the regularization parameter. From the results of numerical simulations, it seems clear that the proposed method works well for the model problem with small measurement error.

Competing interests

The authors declare that they have no competing interests.

Authors' contributions

The authors contributed equally to the writing of this paper. All authors read and approved the final manuscript.

\section{Author details}

'School of Mathematics and Statistics, Northeastern University at Qinhuangdao, Qinhuangdao, 066004, China. ${ }^{2}$ School of Mathematical Sciences, Heilongjiang University, Harbin, 150080, China.

\section{Acknowledgements}

This work was partially supported by National Natural Science Foundation of China (No. 11271113), the New Century Foundation of Heilongjiang Province (No. 1253-NECT-019) and Science and Technology Innovation Team in Higher Education Institutions of Heilongjiang Province (No. 2014TD005).

Received: 9 July 2015 Accepted: 26 October 2015 Published online: 04 November 2015

\section{References}

1. Arqub, OA, Al-Smadi, M: Numerical algorithm for solving two-point, second-order periodic boundary value problems for mixed integro-differential equations. Appl. Math. Comput. 243, 911-922 (2014)

2. Arqub, OA, Al-Smadi, M, Momani, S, Hayat, T: Numerical solutions of fuzzy differential equations using reproducing kernel Hilbert space method. Soft Comput. (2015). doi:10.1007/s00500-015-1707-4

3. Arqub, OA, Al-Smadi, M, Shawagfeh, N: Solving Fredholm integro-differential equations using reproducing kernel Hilbert space method. Appl. Math. Comput. 219, 8938-8948 (2013)

4. Arqub, OA, Abo-Hammour, Z: Numerical solution of systems of second-order boundary value problems using continuous genetic algorithm. Inf. Sci. 279, 396-415 (2014)

5. Momani, S, Arqub, OA, Hayat, T, Al-Sulami, H: A computational method for solving periodic boundary value problems for integro-differential equations of Fredholm-Volterra type. Appl. Math. Comput. 240, 229-239 (2014)

6. Alpay, D: Reproducing Kernel Spaces and Applications. Birkhäuser, Basel (2003)

7. Hon, YC, Wei, T: A fundamental solution method for inverse heat conduction problem. Eng. Anal. Bound. Elem. 28, 489-495 (2004)

8. Miller, K: Least squares methods for ill-posed problems with a prescribed bound. SIAM J. Math. Anal. 1, 52-74 (1970) 
9. Chen, W, Ye, LJ, Sun, HG: Fractional diffusion equations by the Kansa method. Comput. Math. Appl. 59, 1614-1620 (2010)

10. Yu, ZS, Lin, JZ: Numerical research on the coherent structure in the viscoelastic second-order mixing layers. Appl. Math. Mech. 19, 717-723 (1998)

11. Scher, H, Montroll, EW: Anomalous transit-time dispersion in amorphous solids. Phys. Rev. B 12, 2455-2477 (1975)

12. Szabo, TL, Wu, JR: A model for longitudinal and shear wave propagation in viscoelastic media. J. Acoust. Soc. Am. 107, 2437-2446 (2000)

13. Gorenflo, R, Mainardi, F, Moretti, D, Pagnini, G, Paradisi, P: Discrete random walk models for space-time fractional diffusion. Chem. Phys. 284, 521-541 (2002)

14. Metzler, R, Klafter, J: The random walk's guide to anomalous diffusion: a fractional dynamics approach. Phys. Rep. 339 1-77 (2000)

15. Mendes, RV: A fractional calculus interpretation of the fractional volatility model. Nonlinear Dyn. 55, 395-399 (2009)

16. Benson, DA, Wheatcraft, SW, Meerschaert, MM: The fractional-order governing equation of Lévy motion. Water Resour. Res. 36, 1413-1423 (2000)

17. Mainardi, F, Luchko, Y, Pagnini, G: The fundamental solution of the space-time fractional diffusion equation. Fract. Calc. Appl. Anal. 4, 153-192 (2001)

18. Lin, $\mathrm{YM}, \mathrm{Xu}, \mathrm{CJ}$ : Finite difference/spectral approximations for the time-fractional diffusion equation. J. Comput. Phys. $225,1533-1552$ (2007)

19. Luchko, Y: Some uniqueness and existence results for the initial-boundary-value problems for the generalized time-fractional diffusion equation. Comput. Math. Appl. 59, 1766-1772 (2010)

20. Mainardi, F, Pagnini, G: The Wright functions as solutions of the time-fractional diffusion equation. Appl. Math. Comput. 141, 51-62 (2003)

21. $L i, X J, X u, C J$ : A space-time spectral method for the time fractional diffusion equation. SIAM J. Numer. Anal. 47, 2108-2131 (2009)

22. Luchko, Y: Maximum principle for the generalized time-fractional diffusion equation. J. Math. Anal. Appl. 351, 218-223 (2009)

23. Murio, DA: Stable numerical solution of a fractional-diffusion inverse heat conduction problem. Comput. Math. Appl. 53, 1492-1501 (2007)

24. Murio, DA: Time fractional IHCP with Caputo fractional derivatives. Comput. Math. Appl. 56, 2371-2381 (2008)

25. Cheng, J, Nakagawa, J, Yamamoto, M, Yamazaki, T: Uniqueness in an inverse problem for one-dimensional fractional diffusion equation. Inverse Probl. 25, 115002 (2009)

26. Cheng, H, Fu, CL: An iteration regularization for a time-fractional inverse diffusion problem. Appl. Math. Model. 36 5642-5649 (2012)

27. Zheng, GH, Wei, T: Spectral regularization method for solving a time-fractional inverse diffusion problem. Appl. Math. Comput. 218, 396-405 (2011)

28. Zheng, GH, Wei, T: A new regularization method for solving a time-fractional inverse diffusion problem. J. Math. Anal. Appl. 378, 418-431 (2011)

29. Podlubny, I: Fractional Differential Equations. Academic Press, San Diego (1999)

\section{Submit your manuscript to a SpringerOpen ${ }^{\circ}$ journal and benefit from:}

- Convenient online submission

Rigorous peer review

- Immediate publication on acceptance

- Open access: articles freely available online

- High visibility within the field

- Retaining the copyright to your article 